\title{
Effect of a cocoa flavonoid-enriched diet on experimental autoimmune arthritis
}

\author{
Sara Ramos-Romero ${ }^{1}$, Francisco J. Pérez-Cano ${ }^{1,2}$, Teresa Pérez-Berezo ${ }^{1}$, Cristina Castellote ${ }^{1,2,3}$, \\ Angels Franch ${ }^{1,2,3}$ and Margarida Castell ${ }^{1,2 *}$ \\ ${ }^{1}$ Departament de Fisiologia, Facultat de Farmàcia, Universitat de Barcelona, Avinguda Joan XXIII s/n, Edifici B, $3{ }^{a}$ planta, \\ o8028 Barcelona, Spain \\ ${ }^{2}$ Institut de Recerca en Nutrició i Seguretat Alimentària (INSA-UB), Universitat de Barcelona, Barcelona, Spain \\ ${ }^{3}$ CIBER Epidemiología y Salud Pública, Barcelona, Spain
}

(Received 6 January 2011 - Revised 20 April 2011 - Accepted 17 May 2011 - First published online 19 July 2011)

\section{Abstract}

Previously we established that a cocoa-enriched diet in young rats reduces specific antibody production and the $\mathrm{T}$ helper (Th) lymphocyte proportion in lymphoid tissues. The aim of the present study was to ascertain the modulatory ability of a cocoa flavonoidenriched diet on collagen-induced arthritis (CIA), which is mediated by anti-collagen autoantibody response and Th lymphocyte activation. Female Louvain (LOU) rats were fed with a cocoa-enriched diet, beginning 2 weeks before CIA induction. Hind-paw swelling and serum cytokine and anti-collagen antibody concentrations were determined. Anti-collagen antibody-secreting cell counts and lymphocyte subset proportions were established in inguinal lymph nodes (ILN). Reactive oxygen species (ROS), nitric oxide (NO) and TNF $\alpha$ produced by peritoneal macrophages were determined. Although arthritic cocoa-fed rats showed a similar hind-paw swelling time course as the arthritic animals fed a standard diet, the cocoa intake was able to decrease specific IgG2a, IgG2b and IgG2c titres. Moreover, cocoa intake in CIA rats reduced ROS production, TNF $\alpha$ and NO release from peritoneal macrophages, and decreased the Th:cytotoxic T cell ratio in ILN. In conclusion, a cocoa flavonoid-enriched diet in LOU rats with CIA produced no effect on hind-paw swelling but was able to modulate the specific antibody response and also the Th lymphocyte proportion, as well as the synthesis of pro-inflammatory mediators from peritoneal macrophages. Therefore, a cocoa-enriched diet could be a good adjuvant therapy in disorders with oxidative stress or autoimmune pathogenesis.

Key words: Collagen-induced arthritis: Specific antibodies: Oxidative stress: Cocoa flavonoids: Lymphocyte subsets

Rheumatoid arthritis (RA) is a symmetric, polyarticular, systemic and autoimmune inflammatory disease that affects approximately $1 \%$ of the adult population around the world. Multiple factors, including genetic, immune and environmental ones, lead to the progression of this autoimmune disease ${ }^{(1)}$. Among the several environmental factors that contribute to the risk of this disease are smoking, pollution and infections. In contrast, $n-3$ fatty acids, vitamins $\mathrm{D}$ and $\mathrm{K}$, and antioxidants included in the diet are protective compounds against RA ${ }^{(2)}$.

The cells bearing RA-predisposing human leucocyte antigen class II molecules present arthritogenic peptides to $\mathrm{T}$ helper (Th) cells, which become activated cells. These lymphocytes stimulate the production of pro-inflammatory cytokines, especially TNF $\alpha$, IL-1 and IL-6, by monocytes, macrophages and fibroblasts. The synthesis of these cytokines is up-regulated by environmental factors such as smoking and pollution. Activated Th lymphocytes in RA also stimulate B cells to produce autoantibodies. Among these autoantibodies are rheumatoid factor and those that recognise post-translationally citrullinated peptides $^{(3)}$. Although their prevalence is unknown, anti-collagen type II (CII) antibodies have also been identified in serum from RA patients. ${ }^{(3)}$.

Animal models of RA are useful tools for investigating the underlying mechanism of the disease and for the screening of new therapeutic approaches. Type II collagen-induced arthritis (CIA) shares immunological and pathological characteristics with human $\mathrm{RA}^{(4)}$. Genetically susceptible murine strains such as DBA/1, B10.Q and B10.RIII mice or DA and Louvain (LOU) rats are commonly chosen for this RA animal model ${ }^{(4,5)}$ $\mathrm{CIA}$ requires $\mathrm{T}$ - and $\mathrm{B}$-cell response to autologous $\mathrm{CII}^{(6)}$.

Abbreviations: CIA, collagen-induced arthritis; CII, collagen type II; FBS, fetal bovine serum; GM-CSF, granulocyte macrophage colony-stimulating factor; IFN $\gamma$, interferon- $\gamma$; ILN, inguinal lymph nodes; LOU, Louvain; LPS, lipopolysaccharide; mAb, monoclonal antibodies; MCP-1, monocyte chemoattractant protein-1; NO, nitric oxide; RA, rheumatoid arthritis; REF, reference; ROS, reactive oxygen species; SC, secreting cells; Tc, cytotoxic T cell; Th, helper T cell; Treg, regulatory Th cell.

*Corresponding author: M. Castell, fax +34934035901 email margaridacastell@ub.edu 
Th1 cells have been identified in lymphoid tissues following the induction with CII in mice, whereas Th1 cytokines are largely secreted ${ }^{(7)}$. In addition, B cells from CIA animals produce a strong specific immune response against triple helical epitopes of $\mathrm{CII}^{(8)}$. Anti-CII autoantibodies appear to be the primary mechanism of immunopathogenesis in this experimental model. These autoantibodies bind to the joint cartilage, activate the complement cascade and mediate the inflammatory attack to the joints in the CIA model ${ }^{(4,5)}$. Thus, the synergy of humoral and cellular immune response to CII is pivotal for the pathogenesis of this model in susceptible animal strains.

The identification of common dietary substances capable of preventing or modulating RA may have important human health implications. Flavonoids are polyphenols found in fruits, vegetables, tea, wine and cocoa and have ascribed anti-inflammatory properties. These properties are attributed to their antioxidant power as well as their interaction with enzymes implicated in signalling cascades and in the expression of pro-inflammatory genes ${ }^{(9)}$. Flavonoids also modulate pro-inflammatory cytokine production, arachidonic acid metabolism, NADPH oxidase activity and $\operatorname{PPAR}^{(9)}$. One source with a relatively high flavonoid content is cocoa, the product of Theobroma cacao beans ${ }^{(10)}$. In vitro studies have shown the anti-inflammatory properties of cocoa flavonoids in lipopolysaccharide (LPS)-stimulated macrophages ${ }^{(11,12)}$. In addition, cocoa intake in rats decreases the local hind-paw swelling induced by carrageenin or bradykinin and also reduces $\mathrm{TNF} \alpha$ concentration in inflammatory exudates ${ }^{(13,14)}$. In addition, an extended cocoa-enriched diet diminishes specific antibody production in immunised rats ${ }^{(15)}$, and also reduces the Th lymphocyte proportion in the spleen and lymph nodes in young rats ${ }^{(16)}$. Owing to the fact that CIA pathogenesis requires Th cells and a specific antibody response, we can hypothesise that a cocoa-enriched diet could modulate this autoimmune model.

The aim of the present study was to assess whether a cocoaenriched diet could reduce the anti-CII autoantibody response and also down-regulate Th activation in rat CIA, therefore modulating the inflammatory response.

\section{Materials and methods}

\section{Animals}

Female LOU/CNimrOlaHsd rats, aged 7-9 weeks, were obtained from Harlan (Barcelona, Spain). The rats were housed three or four per cage in controlled conditions of temperature $\left(20 \pm 2^{\circ} \mathrm{C}\right)$, humidity $(55 \%)$ and $12 \mathrm{~h}$ light $-12 \mathrm{~h}$ dark cycle conditions, with food and water ad libitum. The design and the number of animals per group, obeying the institutional guidelines for the care and use of laboratory animals, were approved by the Ethics Committee for Animal Experimentation at the University of Barcelona and the Catalonian government.

\section{Experimental design}

Animals were randomly distributed into four different experimental groups (ten rats per group) called reference (REF),
Cocoa, CIA and CIA-Cocoa. The REF and CIA groups were fed with a standard diet formulated following the American Institute of Nutrition (AIN) indications that provides the nutrients required for optimal rat maintenance (AIN-93M diet) ${ }^{(17)}$. The Cocoa and CIA-Cocoa groups were fed with a diet containing cocoa, which was prepared by adding cocoa to a modified AIN-93M diet in which maize starch, soyabean oil, cellulose and casein had been partially removed. The resulting cocoa diet had the same proportion of carbohydrates, lipids, proteins and total energy as the standard diet. Cocoa was provided by Nutrexpa (Barcelona, Spain) and contained $22 \%$ proteins, $16 \%$ carbohydrates, $11 \%$ lipids and $25.5 \%$ fibre, and $10.62 \mathrm{mg}$ of total phenols per g (Folin-Ciocalteu). Animals were fed with a $10 \%$ cocoa diet for $14 \mathrm{~d}$ before CIA induction and during the latency period ( $14 \mathrm{~d}$ after induction), and thereafter with a $5 \%$ cocoa diet until the end of the study.

\section{Induction and assessment of collagen-induced arthritis}

The induction of CIA in rats was based on the method described by Brahn et al. ${ }^{(18)}$. Type II bovine collagen (MD Biosciences, Zurich, Switzerland) was dissolved in cold $0.05 \mathrm{M}$-acetic acid $(2 \mathrm{mg} / \mathrm{ml})$ and emulsified with an equal volume of incomplete Freund's adjuvant (Sigma-Aldrich, St Louis, MO, USA). The CIA and CIA-Cocoa rats were injected intradermally into the lower back with $0.2 \mathrm{ml}$ of this collagen emulsion. Arthritis was clinically assessed, in a blinded manner, by means of hind-paw volume measure with a water plethysmometer (7140; Ugo Basile, Comerio, Italy). Left and right hind-paw volumes were measured on day 0 (before CIA induction), on day 7 (post-induction) and afterwards every other day until day 27 (post-induction). Articular swelling was expressed as the addition of right and left hindpaw volume increase percentages with respect to their volume on day 0 .

\section{Sample collection and processing}

During the present study, blood samples were collected from the saphenous vein to determine anti-CII antibody concentration in sera. On day 27 after induction, peritoneal macrophages and inguinal lymph nodes (ILN) were obtained.

Peritoneal macrophages from CIA animals were collected by injecting $40 \mathrm{ml}$ ice-cold sterile PBS ( $\mathrm{pH} 7 \cdot 2$ ) into the peritoneal cavity. After abdominal massage, cell suspension was aspirated, centrifuged and resuspended in cold DMEM + GlutaMAX media (Invitrogen, Paisley, UK) containing 10\% fetal bovine serum (FBS; PAA, Pasching, Austria), and 100 $\mathrm{IU} / \mathrm{ml}$ streptomycin-penicillin (Sigma; DMEM + G-FBS).

ILN were broken up by passing through a nylon cell strainer (BD Biosciences, Erembodegem, Belgium) with RPMI 1640 media (PAA) containing 10\% FBS. Cells were then centrifuged and resuspended in PBS.

The number and viability of peritoneal macrophages and ILN lymphocytes were determined by acridine orange and ethidium bromide (Sigma) staining followed by fluorescence light microscopical analysis. 


\section{Quantification of serum anti-collagen type II antibodies by ELISA}

The concentration of serum total, IgM, IgG1, IgG2a, IgG2b and IgG2c anti-CII antibodies was determined by ELISA. Polystyrene microELISA plates (Nunc Maxisorp, Wiesbaden, Germany) were incubated with CII solution $\left(1.25 \mu \mathrm{g} / \mathrm{ml} \mathrm{CII} \mathrm{in} \mathrm{PBS,} 4^{\circ} \mathrm{C}\right.$, overnight), obtained by stirring CII overnight at $4^{\circ} \mathrm{C}$ in $0 \cdot 1 \mathrm{M}-$ acetic acid, and a $24 \mathrm{~h}$ dialysis against PBS. After CII coating, serum samples were incubated ( $3 \mathrm{~h}$, room temperature). To measure total anti-CII antibody concentration, we used a peroxidase-conjugated goat anti-rat Ig (BD Biosciences) as the detection antibody. For specific isotypes, biotin-conjugated anti-rat IgM, IgG1, IgG2a, IgG2b or IgG2c monoclonal antibodies (mAb; BD Biosciences) were applied, followed by extravidin-peroxidase (Sigma) incubation. Lastly, an $o$-phenylenediamine (Sigma) solution with $\mathrm{H}_{2} \mathrm{O}_{2}(0 \cdot 04 \%)$ was added. Absorbance was measured at $492 \mathrm{~nm}$ after stopping reaction (3 $\mathrm{M}-\mathrm{H}_{2} \mathrm{SO}_{4}$ ). Since standards were not available, several dilutions of pooled sera from the CIA animals were added to each plate. Taking into account the required dilution of samples, this pool was arbitrarily assigned as $40000 \mathrm{U} / \mathrm{ml}$ total; $32000 \mathrm{U} / \mathrm{ml} \mathrm{IgG2a;} 2000 \mathrm{U} / \mathrm{ml}$ IgG1 and IgG2b; $500 \mathrm{U} / \mathrm{ml}$ IgG2c; and $250 \mathrm{U} / \mathrm{ml}$ IgM anti-CII antibodies.

\section{Quantification of anti-collagen type // antibody-secreting cells by ELISPOT}

Anti-CII IgM- and IgG-secreting cells (SC) from ILN were enumerated by the ELISPOT technique, as described by Pérez-Berezo et al. ${ }^{(15)}$, with some modifications. A ninety-six-well hydrophobic PVDF plate (Multiscreen, catalogue no. MAIPS4510; Millipore, Eschborn, Germany) was pre-wet with 35\% ethanol, washed with PBS and coated with CII solution $(20 \mu \mathrm{g} / \mathrm{ml}$, overnight at $\left.4^{\circ} \mathrm{C}\right)$. ILN lymphocytes $\left(20-10-5-2.5 \times 10^{4}\right.$ cells per well) were incubated for $24 \mathrm{~h}$. Biotin-conjugated goat anti-rat IgM or IgG antibodies were added followed by extravidinperoxidase conjugate. Spots were visualised by the addition of 3-amino-9-ethylcarbazole plus $\mathrm{H}_{2} \mathrm{O}_{2}$, counted automatically by the computer-assisted ELISPOT image analysis (ELISPOT Reader System; AID, Strassberg, Germany) and expressed as number of anti-CII antibody-SC per $10^{6}$ cells.

\section{Inguinal lymph nodes lymphocyte subsets by immunofluorescence staining and flow cytometry}

ILN cell phenotype was determined by simple, double or triple staining, followed by flow cytometry analysis. Mouse anti-rat mAb conjugated to fluorescein isothiocyanate, phycoerythrin, peridinin chlorophyll protein or allophycocyanin used here included anti- $\alpha \beta$ TCR (R73), anti- $\gamma \delta$ TCR (V65), anti-CD4 (OX-35), anti-CD25 (IL-2R $\alpha$ chain, OX-39), antiCD8 $\alpha$ (OX-8), anti-CD45RA (OX-33, CD45 isoform only on rat $\mathrm{B}$ cells; $\mathrm{BD}$ Biosciences), and anti-Foxp3 (FJK-16a; eBioscience, Frankfurt, Germany).

Extracellular staining was performed in $5 \times 10^{5}$ cells by saturating concentrations of fluorochrome-conjugated $\mathrm{mAb}$ in PBS containing $1 \% \mathrm{FBS}$ and $0 \cdot 1 \% \mathrm{NaN}_{3}\left(30 \mathrm{~min}, 4^{\circ} \mathrm{C}\right.$, in darkness). A negative control staining using an isotypematched $\mathrm{mAb}$ was included for each sample. For intracellular staining, $5 \times 10^{5}$ cells previously labelled with anti-CD4phycoerythrin and anti-CD25-fluorescein isothiocyanate mAb were treated with a Foxp3 Fixation/Permeabilisation Kit (eBioscience). Then, intracellular staining with anti-Foxp3allophycocyanin mAb was carried out. Analysis was performed using a Cytomics FC500-MPL cytometer (Beckman Coulter, Miami, FL, USA). Lymphocyte subsets were defined in the cytometer as $\mathrm{T} \alpha \beta(\mathrm{TCR} \alpha \beta+), \operatorname{T} \gamma \delta(\mathrm{CD} 8+\mathrm{TCR} \gamma \delta+), \mathrm{B}$ $(\mathrm{CD} 45 \mathrm{RA}+)$, Th $(\mathrm{CD} 4+\mathrm{TCR} \alpha \beta+)$, T cytotoxic $(\mathrm{Tc} ; \mathrm{TCR} \alpha \beta+$ $\mathrm{CD} 8 \alpha+)$, activated Th $(\mathrm{CD} 4+\mathrm{CD} 25+$ Foxp3-) and regulatory Th (Treg; CD $4+\mathrm{CD} 25+$ Foxp3 + ). Results are expressed as a percentage of positive cells in the lymphocyte population, previously selected according to the forward and side scatter characteristics. In some cases, results are shown as a percentage of positive cells in a specific lymphocyte subset (Th and Tc in T cells; activated Th and Treg in Th cells).

\section{Determination of serum cytokine concentration by flow cytometry}

IL-1 $\alpha$, monocyte chemoattractant protein-1 (MCP-1), TNF $\alpha$, interferon- $\gamma$ (IFN $\gamma$ ) and granulocyte macrophage colony-stimulating factor (GM-CSF) were quantified in the sera at day 27 after induction. For this purpose, a rat cytokine multiplex kit (Bender MedSystems GmbH, Vienna, Austria) was used following the manufacturer's instructions. Analysis was performed using a Cytomics FC500-MPL cytometer (Beckman Coulter) and the FlowCytomix Pro2.2.1 program from Bender MedSystems GmbH.

\section{Reactive oxygen species production from peritoneal macrophages by dichlorofluorescein assay}

Peritoneal macrophages $\left(2.5 \times 10^{4}\right.$ cells per $100 \mu \mathrm{l}$ in DMEM + GFBS) were plated in ninety-six-well black plates (Corning Incorporated Life Sciences, Lowell, MA, USA) and allowed to attach overnight. Macrophages were washed once with warm DMEM medium without phenol red (Sigma) and incubated with 20 $\mu \mathrm{mol} / 1$ of reduced $2^{\prime}, 7^{\prime}$-dichlorofluorescein diacetate probe $\left(\mathrm{H}_{2} \mathrm{DCF}-\mathrm{DA}\right.$; Invitrogen) for $30 \mathrm{~min}$ at $37^{\circ} \mathrm{C}$. Fluorescence, directly proportional to reactive oxygen species (ROS) production, was measured every $30 \mathrm{~min}$ by fluorometry (excitation $538 \mathrm{~nm}$, emission $485 \mathrm{~nm}$ ) up to $2 \mathrm{~h}$. For each animal, the background from corresponding cells without $\mathrm{H}_{2}$ DCF-DA was subtracted.

\section{Quantification of TNF $\alpha$ and nitric oxide from peritoneal macrophages}

Peritoneal macrophages $\left(1.2 \times 10^{6}\right.$ cells per $1 \mathrm{ml}$ in DMEM + G-FBS) were plated in twelve-well flat-bottom plates and cultured overnight to allow macrophage adhesion. After washing with warm sterile PBS, macrophages were stimulated by an addition of $1 \mu \mathrm{g} / \mathrm{ml}$ LPS from Escherichia coli (O55:B5; Sigma) or were kept non-stimulated. After $24 \mathrm{~h}$, supernatants were collected and stored at $-80^{\circ} \mathrm{C}$.

The concentration of TNF $\alpha$ in supernatants was determined using a rat ELISA set (BD Biosciences) following the manufacturer's 
(a)

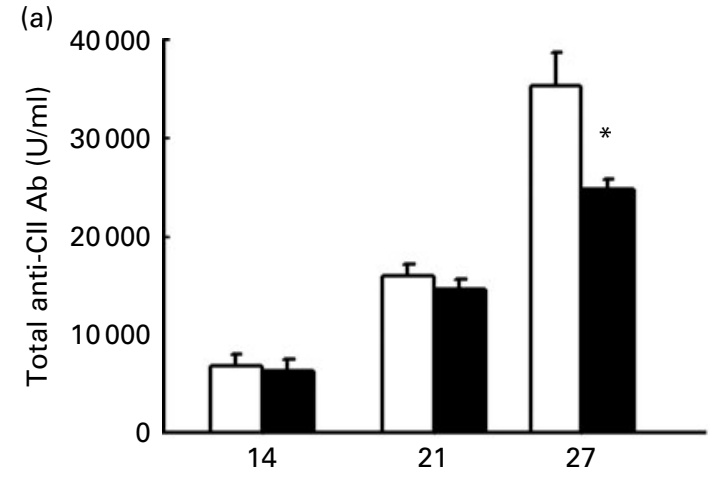

(c)
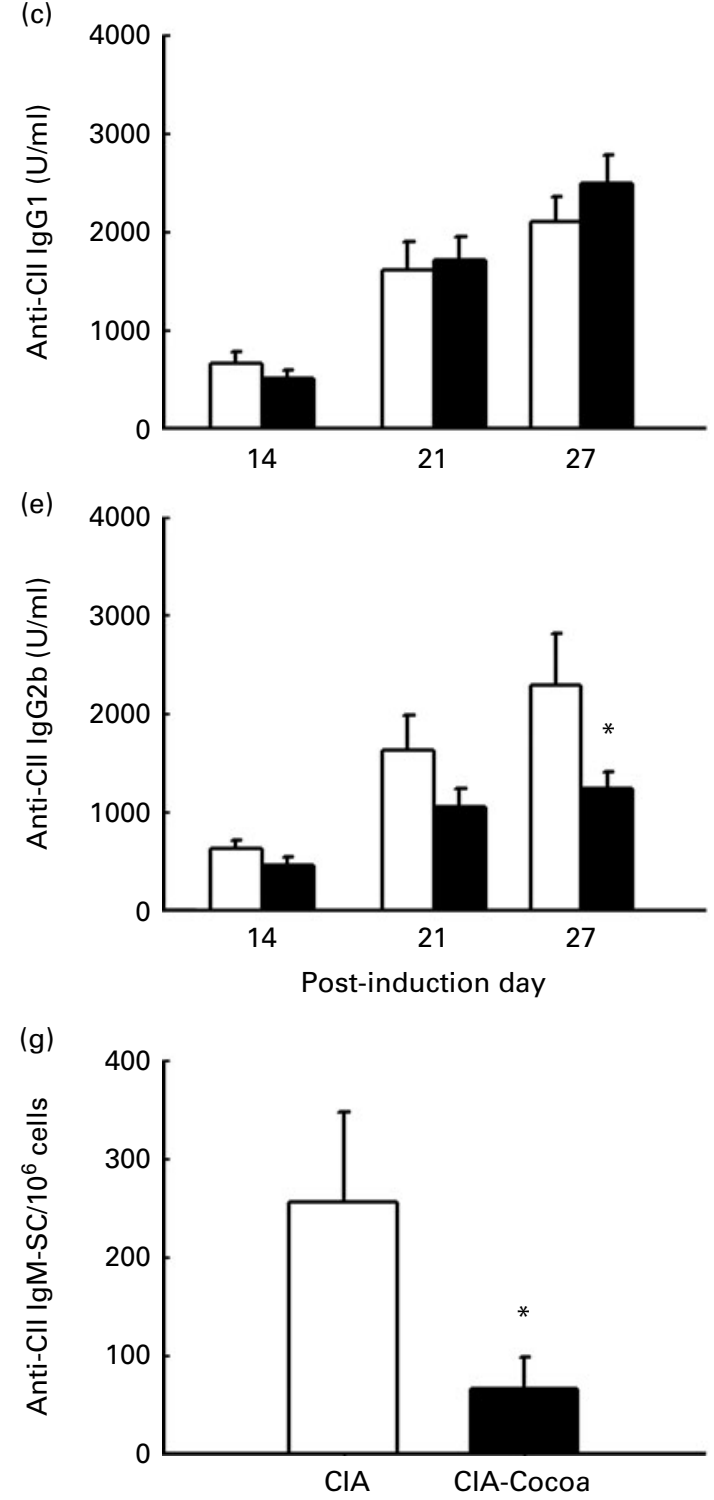
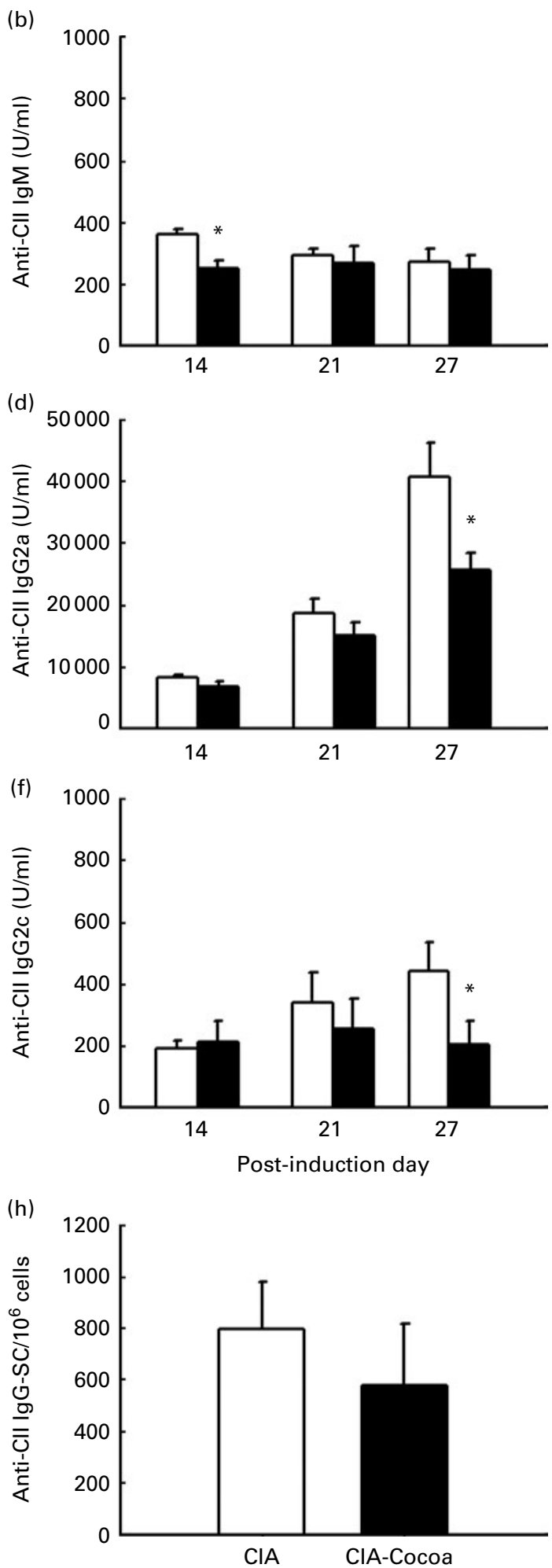

Fig. 1. Serum anti-collagen type II antibody concentration and anti-collagen type II antibody-secreting cell counts in inguinal lymph nodes (ILN). Serum anticollagen type II (CII; a) total antibodies, (b) IgM, (c) IgG1, (d) IgG2a, (e) IgG2b and (f) IgG2c concentrations during the study. Anti-CII (g) IgM-, and (h) IgG-SC from ILN at day 27 post-arthritis induction. Values are means, with their standard errors represented by vertical bars $(n+10)$. ${ }^{*}$ Mean values were significantly different from those of the collagen-induced arthritis (CIA; $\square)$ group $(P<0.05$; ANOVA). $\mathbf{\square}$, CIA-Cocoa. 
instructions. The stable end product of nitric oxide (NO), nitrite ion, was quantified by a modification of the Griess reaction.

\section{Statistics}

The software package SPSS 16.0 (SPSS, Inc., Chicago, IL, USA) was used for statistical analysis. Conventional one-way ANOVA was performed, taking the experimental group as the independent variable. When diet had a significant effect on the dependent variable, the Bonferroni correction for multiple comparisons was applied. Significant differences were accepted when $P<0 \cdot 05$.

\section{Results}

\section{Effect of cocoa diet on serum anti-collagen type II} antibodies in collagen-induced arthritis rats

CIA involved the synthesis of antibodies directed against CII, which increased progressively during the study period (Fig. 1). Anti-CII antibodies mainly belonged to the IgG2a isotype. The cocoa diet reduced anti-CII antibody concentration at day 27 after induction (Fig. 1(a); $P<0 \cdot 01$ ). A deeper study of anti-CII antibodies revealed that the effect of the cocoa diet was different according to the antibody isotype. Anti-CII IgM antibodies were reduced by the cocoa diet only in the early phase (Fig. 1(b); $P<0.05$ on day 14). Anti-CII IgG2a, IgG2b and IgG2c antibody titres were also diminished by the cocoa diet, mainly in the late arthritic phase (Fig. 1(d)-(f); $P<0.05$ on day 27).

Anti-CII antibody-SC in ILN were quantified at day 27 after induction (Fig. 1(g) and (h)). Although the anti-CII IgG-SC number was not significantly modified by cocoa diet in ILN, anti-CII IgMSC counts were significantly diminished (Fig. 1(g); $P<0.05)$.

Effect of cocoa diet on inguinal lymph nodes lymphocyte subsets

The study of the main lymphocyte populations in ILN at day 27 after induction (Fig. 2(a)) did not show dramatic changes but revealed that the two groups that were fed the cocoa diet decreased the percentage of T $\alpha \beta$ cells $(P<0 \cdot 05)$. Regarding the T-cell subsets, CIA involved changes in the proportion of Th and Tc cells, by reducing the Th cell proportion and increasing the Tc percentage (Fig. 2(b); $P<0.05$ when comparing the CIA group against the REF group). The cocoa diet also decreased the Th cell percentage and increased the Tc lymphocyte proportion in ILN $(P<0.05$ when comparing Cocoa group against the REF group). In CIA-Cocoa animals, changes due to CIA and the cocoa diet were added and the Th:Tc ratio was lower than that in the CIA group $(P<0.05$ CIA-Cocoa group against both REF and CIA groups).

At 4 weeks after CIA induction, the proportion of Treg in Th cells was significantly increased in both CIA and CIA-Cocoa groups (Fig. 2(c); $P<0 \cdot 05$ ).
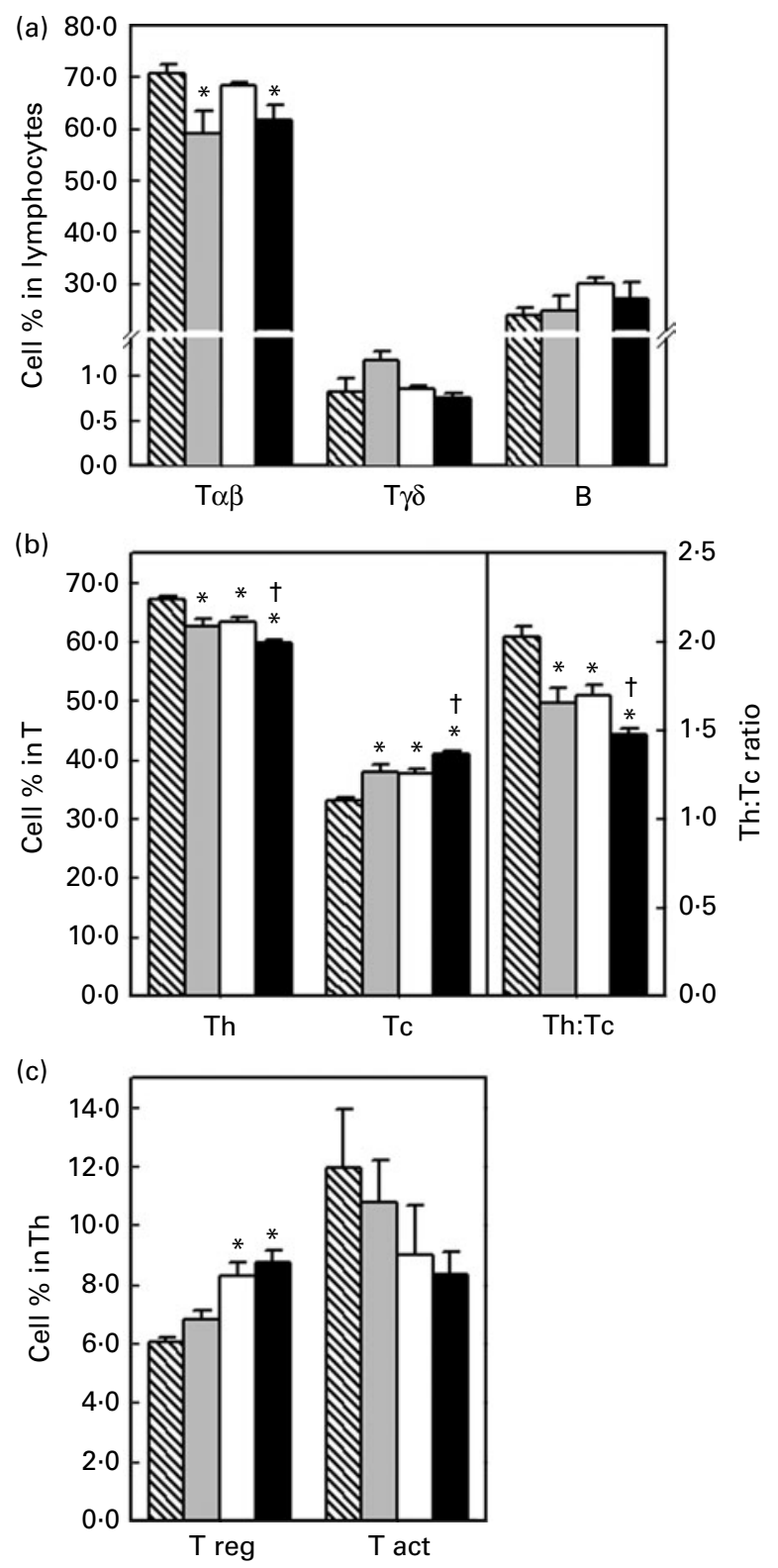

Fig. 2. Lymphocyte subset composition in rat inguinal lymph nodes. (a) $T \alpha \beta$, $T \gamma \delta$ and B-cell percentages with respect to lymphocytes. (b) T helper (Th) and $\mathrm{T}$ cytotoxic (Tc) cell proportions in the $\mathrm{T}$ population, and Th:Tc ratio. (c) Regulatory Th (Treg) and activated Th (T act) cell percentages in the Th subset. Values are means, with their standard errors represented by vertical bars $(n 5-9)$. * Mean values were significantly different from those of the reference $(\mathrm{REF})(\mathbb{\mathbb { S }})$ group $(P<0.05$; ANOVA followed by Bonferroni correction). † Mean values were significantly different from those of the collageninduced arthritis (CIA; $\square)$ group $(P<0.05$; ANOVA followed by Bonferroni correction). $\square$, Cocoa; $\square$, ClA-Cocoa.

\section{Effect of cocoa diet on articular swelling}

On day 12 after CIA induction, some animals started to show articular swelling (Fig. 3). The number of arthritic animals and the hind-paw volume increased progressively until day 19 after induction. Thereafter, hind-paw swelling tended to decrease. The CIA and CIA-Cocoa groups showed the same pattern of hind-paw inflammation. 


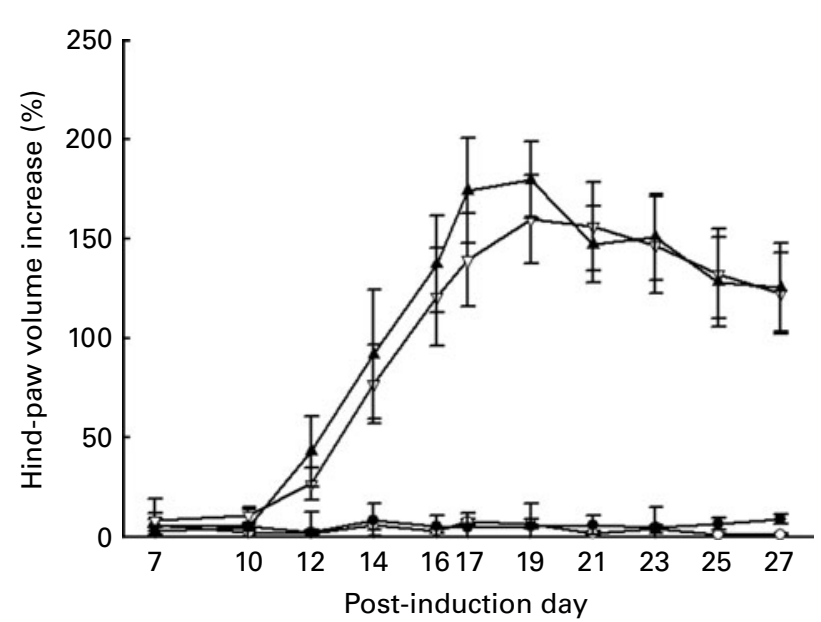

Fig. 3. Hind-paw volume increase over 4 weeks from arthritis induction (expressed as the addition of right and left hind-paw increase percentages with respect to day 0 ). Values are means, with their standard errors represented by vertical bars ( $n$ 7-10; ANOVA followed by Bonferroni correction). -O-, Reference REF; - - -, Cocoa; $-\nabla-$, collagen-induced arthritis (CIA); - $\mathbf{-}$, ClA-Cocoa.

\section{Effect of cocoa diet on serum cytokines}

Serum IL- $1 \alpha$, MCP-1, TNF $\alpha$, IFN $\gamma$ and GM-CSF concentrations were measured at day 27 after induction (Table 1). Among the cytokines considered, MCP-1 was the most abundant in the rat sera of the four experimental groups, and the cocoa diet in the healthy animals reduced its concentration $(P<0.05)$. At 4 weeks after CIA induction, cytokine values were very low and no differences were found between the REF and CIA groups. The cocoa diet in the arthritic animals (CIA-Cocoa group) produced an increase in the serum concentrations of TNF $\alpha$, IFN $\gamma$ and GM-CSF compared to those of the CIA and REF groups $(P<0 \cdot 05)$.

\section{Effect of cocoa diet on inflammatory mediators by} peritoneal macrophages of collagen-induced arthritis rats

Ex vivo ROS production by peritoneal macrophages from the CIA and CIA-Cocoa groups was measured over $2 \mathrm{~h}$ (Fig. 4(a)). Macrophages isolated from the CIA animals synthesised higher amounts of ROS than those from the CIACocoa group after just $30 \mathrm{~min}$ and this continued during the study period $(P<0.05)$.
TNF $\alpha$ and NO production were quantified in macrophage supernatants that were obtained $24 \mathrm{~h}$ after LPS stimulation and in resting conditions (Fig. 4(b) and (c)). TNF $\alpha$ secretion by CIA-Cocoa macrophages was lower than that in the CIA group $(P<0.05$ in LPS-stimulated cells; Fig. 1(b)). Similarly, NO production from LPS-stimulated macrophages in the CIA-Cocoa group was lower than that in the CIA macrophages $(P<0.05$ in LPS-stimulated cells; Fig. $4(\mathrm{c}))$ and was similar to that produced in resting conditions. Viability of macrophages after $24 \mathrm{~h}$ culture was similar in both the groups (data not shown).

\section{Discussion}

The present study shows that a cocoa-enriched diet during rat CIA was able to reduce the synthesis of specific antibodies against type II collagen, to decrease the Th lymphocyte proportion in ILN and to reduce the release of inflammatory mediators from peritoneal macrophages. However, these immunomodulatory effects were not enough to reduce the hind-paw swelling in arthritic animals during the study period.

Anti-CII autoantibodies have been described as the cornerstone in CIA pathogenesis ${ }^{(19,20)}$, and some effective treatments of CIA in rats were accompanied by a reduction of this autoimmune response ${ }^{(21-24)}$. In the present study, we found a decrease in serum anti-CII antibody concentrations in cocoafed CIA animals, although this reduction did not lead to improvement in the hind-paw swelling during the study period. We should take into account the fact that the decrease in anti-CII antibody concentration was only observed at the end of the study, and it could be that this down-regulatory effect of cocoa would only be reflected on paw inflammation over a longer study. The cocoa diet reduced anti-CII antibody titres in CIA LOU rats to a lesser extent and more slowly than we expected. In previous studies, we observed that cocoa intake decreased serum IgG and IgM concentrations in healthy young Wistar rats ${ }^{(25)}$, as well as the antigen-specific antibody titres after 4 weeks of immunisation in adult Wistar rats ${ }^{(15)}$. Moreover, cocoa intake reduced the serum antimycobacteria antibody concentration in adult Wistar rats after 2 weeks of immunisation $^{(26)}$. As LOU rats fed with cocoa did not show a decrease in anti-CII antibody response at 2 weeks of CIA induction, this could suggest that the rat strain is a determining factor for the immunomodulatory action of cocoa. On the

Table 1. Cytokine concentration in sera at day 27 post-arthritis induction

(Mean values with their standard error)

\begin{tabular}{|c|c|c|c|c|c|c|c|c|c|c|}
\hline & \multicolumn{2}{|c|}{$\mathrm{IL}-1 \alpha(\mathrm{pg} / \mathrm{ml})$} & \multicolumn{2}{|c|}{ MCP-1 (pg/ml) } & \multicolumn{2}{|c|}{$\mathrm{TNF} \alpha(\mathrm{pg} / \mathrm{ml})$} & \multicolumn{2}{|c|}{$\mathrm{IFN} \gamma(\mathrm{pg} / \mathrm{ml})$} & \multicolumn{2}{|c|}{ GM-CSF (pg/ml) } \\
\hline & Mean & SEM & Mean & SEM & Mean & SEM & Mean & SEM & Mean & SEM \\
\hline $\operatorname{REF}(n 7)$ & $6 \cdot 41$ & 2.03 & 93.81 & 5.9 & 1.92 & 0.23 & 1.44 & 0.06 & $8 \cdot 87$ & 0.88 \\
\hline Cocoa $(n 6)$ & 3.71 & 0.67 & $50 \cdot 34^{*}$ & $9 \cdot 64$ & $1 \cdot 76$ & 0.43 & 1.26 & 0.15 & $7 \cdot 66$ & 1.34 \\
\hline $\mathrm{CIA}(n 6)$ & 5.08 & 1.75 & 103.49 & $14 \cdot 23$ & 1.58 & 0.26 & 1.41 & 0.15 & $7 \cdot 80$ & 1.00 \\
\hline CIA-Cocoa $(n 10)$ & 11.33 & $5 \cdot 20$ & $104 \cdot 02$ & $14 \cdot 71$ & $3 \cdot 23^{* \prime} \dagger$ & 0.48 & $1.87^{\star *} \dagger$ & 0.12 & $14 \cdot 60^{*} \dagger$ & 1.88 \\
\hline
\end{tabular}

MCP-1, monocyte chemoattractant protein-1; IFN $\gamma$, interferon- $\gamma$; GM-CSF, granulocyte macrophage colony-stimulating factor; REF, reference; CIA,

* Mean values were significantly different from that of the REF group $(P<0.05$; one-way ANOVA followed by Bonferroni correction).

$\dagger$ Mean values were significantly different from that of the CIA group $(P<0.05$; one-way ANOVA followed by Bonferroni correction). 

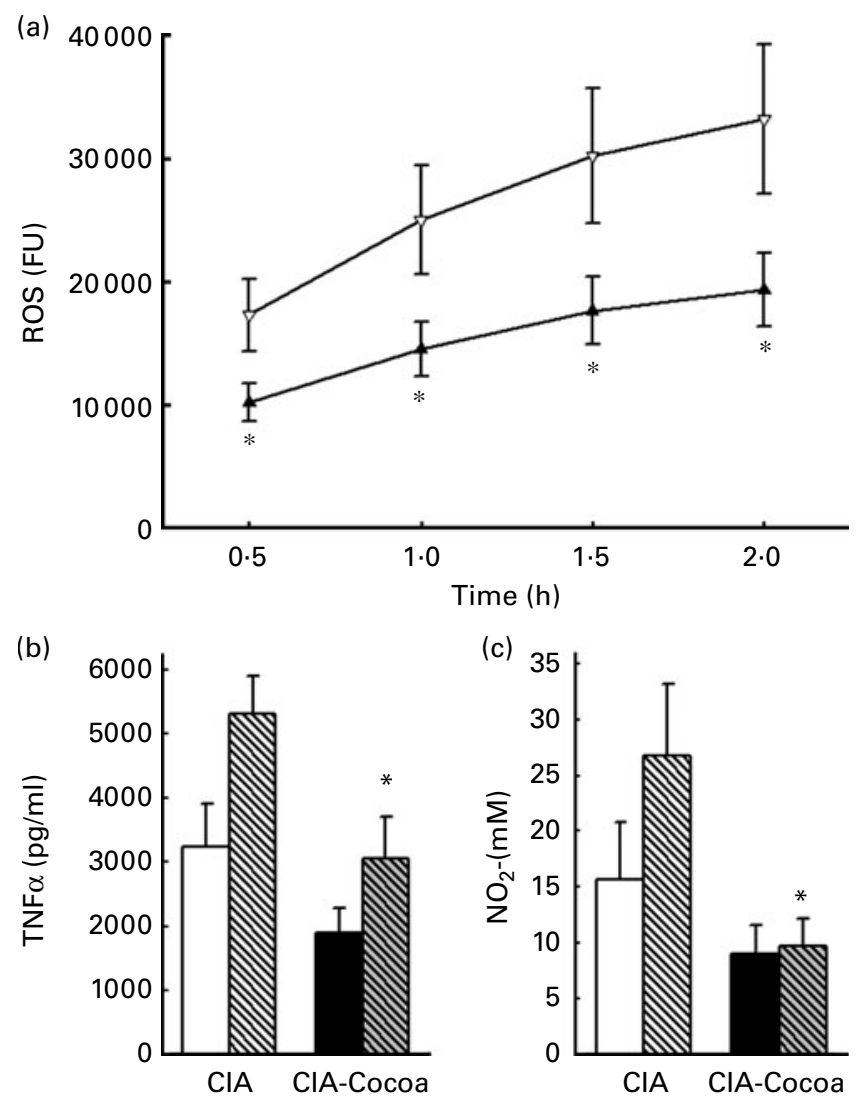

Fig. 4. Reactive oxygen species (ROS), TNF $\alpha$ and nitric oxide (NO) production by peritoneal macrophages of the collagen-induced arthritis ( $\mathrm{ClA}$; $\neg-$ ) and CIA-Cocoa ( - ) groups. (a) Time course of ROS production expressed in fluorescence units (FU) determined by dichlorofluorescein assay. (b) TNF $\alpha$ concentration in cell culture supernatants determined by ELISA in lipopolysaccharide (LPS; $\mathbb{\nabla}$ )-stimulated and non-stimulated (NS; $\square$ ) macrophages. (c) NO cell production, quantified as nitrite ion $\left(\mathrm{NO}_{2}^{-}\right)$concentration, measured by Griess assay in LPS-stimulated and resting conditions. Values are means, with their standard errors represented by vertical bars $(n$ 7-10). * Mean values were significantly different from those of collagen-induced arthritis $(\mathrm{CIA})$ group $(P<0.05$; ANOVA).

other hand, it is interesting to consider the differential cocoa influence on anti-CII antibody isotypes. The present study found that in the CIA rats, anti-CII IgG2a antibodies were the most abundant in sera, which concurs with other studies $^{(27-29)}$. Cocoa intake produced an early decrease in anti-CII IgM values and, at a later stage, a reduction in the specific anti-CII IgG2a antibodies, together with a decrease in anti-CII IgG2b and IgG2c concentrations, without affecting IgG1 specific antibodies. Some strategies modulating CIA in rats have reported a reduction in anti-CII IgG2a and IgG2b isotypes, whereas the anti-CII IgG1 remained or increased ${ }^{(22-24)}$. While IgG2b is clearly considered to be a Th1-related antibody, the role of IgG2a antibodies in rats is controversial. Some authors ${ }^{(30,31)}$ have demonstrated IgG2a properties as Th2-related antibodies, but other authors ${ }^{(22,24)}$ consider that, as in mice, IgG2a is a Th1-related antibody. In any case, the down-regulation of anti-CII IgG2a, IgG2b and IgG2c autoantibodies, together with the maintenance of $\operatorname{IgG} 1$ proportion, did not improve the hind-paw swelling at the end of the present study. It is still unknown whether this effect could be subsequently beneficial. On the other hand, we enumerated the anti-CII antibody-SC in ILN, anti-CII IgG-SC being more abundant than anti-CII IgM. Cocoa intake only decreased the number of anti-CII IgM-SC in ILN, without modifying the number of anti-CII IgG-SC. These results did not reflect the changes in serum antibody concentrations and suggest that the most abundant antibody-SC would be located in other lymphoid tissues, such as the closer regional popliteal lymph nodes and/or the bone marrow.

Although cocoa did not effectively prevented CIA development, it should be pointed out that the inflammatory potential of the CII immunisation was reduced in cocoa-fed CIA animals because peritoneal macrophages showed less ability to secrete inflammatory mediators, including ROS. These results are in line with our previous studies performed in vitro ${ }^{(12)}$ and with the results of TNF $\alpha$ and NO secretion obtained from LPS-stimulated macrophages isolated from healthy animals fed $\operatorname{cocoa}^{(13,14,32)}$. The present study results demonstrate the antioxidant and anti-inflammatory potential of cocoa in a pathological status after being intestinally absorbed. It has been reported that antioxidants reduce the activation of $\mathrm{NF}-\kappa \mathrm{B}$, which is involved in the production of pro-inflammatory cytokines such as $\mathrm{TNF}^{(33)}{ }^{33}$. In addition, the development of RA has been related to oxidative status induced by smoking or pollution $^{(2)}$, and therefore a diet enriched with antioxidants, such as cocoa flavonoids, could have a role in the prevention of, or acting as adjuvant therapy for, RA. The amount of cocoa intake needed for such beneficial effect in human subjects can be established following the conversion of animal doses into human equivalent doses described by Reagan-Shaw et $a l^{(34)}$. According to this conversion, the $10 \%$ and $5 \%$ cocoa-enriched diets would be equivalent to 0.908 and $0.454 \mathrm{~g}$ cocoa per $\mathrm{kg}$ human per d respectively ( 54.5 and $27 \cdot 25 \mathrm{~g}$ cocoa for a $60 \mathrm{~kg}$ person, respectively). On the other hand, it must be considered that cocoa intake also provides other bioactive compounds such as fibre and methylxanthines like theobromine ${ }^{(35)}$. It has been reported that high concentrations of methylxanthines can suppress inflammation by inhibiting phosphodiesterases ${ }^{(36)}$, and fibre can change the intestinal microbiota, which may influence the intestinal and systemic immune cell function ${ }^{(37)}$.

The present study also shows the effect of CIA and cocoa intake on the lymphocyte composition of ILN. We found that in comparison with healthy animals, ILN from CIA rats contained a reduced Th:Tc ratio and an increased Treg proportion at day 27 post-arthritis induction. These results do not match with the blood increase of the $\mathrm{CD}^{+} / \mathrm{CD}^{+}$ ratio in CIA rats reported by Wang et al. ${ }^{(38)}$. In relation to the cocoa diet, the present study found a decrease in the Th:Tc ratio similar to previous studies ${ }^{(16,25)}$. Initially, we expected that the decrease in Th population induced by a cocoa diet could control this autoimmune disease. However, although Th cells have an important role in CIA pathogenesis ${ }^{(6)}$, arthritic rats fed with standard diet also underwent a reduction in Th proportion at day 27 after induction. Therefore, when considering this feature in CIA animals, it seems logical that there were no beneficial effects to the relative reduction of Th cells induced by the cocoa diet. Nevertheless, 
further functional studies should be performed to clarify the meaning and repercussions of such a relative decrease in Th. In addition, at 4 weeks after arthritis induction, we found an increase in the proportion of the Treg subset in ILN from the CIA and CIA-Cocoa rats. It has been reported that Treg cells regulate the immune responses during the arthritic process because the transference of Treg cells to CIA mice prevents or slows the disease development ${ }^{(39,40)}$ Moreover, a reduced number or defective function of Treg cells have been involved in the pathogenesis of $\mathrm{RA}^{(41)}$. In the present study, a reduced number of Treg in ILN was not found, on the contrary, its proportion increased at 4 weeks of arthritis induction. This result, together with the relative decrease in hind-paw swelling at this time, suggests that the increase in Treg cells in CIA animals could reflect the onset of the recovery phase.

Other results that deserve special attention refer to serum cytokines. We found that the serum concentration of some pro-inflammatory cytokines in CIA rats, such as IL- $1 \alpha$, MCP-1, TNF $\alpha$, IFN $\gamma$ and GM-CSF, was similar to that in healthy animals. These results are in line with other studies determining serum pro-inflammatory cytokines 1 month after induction in CIA rats ${ }^{(42,43)}$, although other studies showed an increase in such cytokines ${ }^{(21,22)}$. CIA rats fed with cocoa showed higher concentrations of TNF $\alpha$, IFN $\gamma$ and GM-CSF than those fed with the standard diet even though they presented a similar hind-paw swelling. All these results showed that there was no correlation between the articular swelling and serum cytokine concentrations, at least in the rat CIA model and at the time of the present study. This lack of correlation could be due to the fact that, at day 27 after induction, the arthritis was not in the active phase of the inflammation. Conversely, it could be that cytokines secreted by cells closer to joint inflammation would be a better indicator of the inflammatory status. Moreover, as the cocoa diet increased some serum inflammatory cytokines, it remains to be clarified why the cocoa diet was able to reduce the inflammatory mediators released by peritoneal macrophages from CIA animals but cells elsewhere did not. The effect of the cocoa diet on the chemokine MCP-1, the most abundant of the five cytokines considered, was particularly notable. Cocoa intake decreased the concentration of MCP-1 in the sera from healthy animals and showed no differences between either group of CIA rats, in contrast to the other studied cytokines. The present results in healthy rats concur with those reported previously in vitro ${ }^{(12)}$. MCP-1 is a chemokine released by macrophages that is involved in cell infiltration and the migration to the joint of monocytes, $\mathrm{T}$ and NK cells. Therefore, the decrease in MCP-1 concentration could reflect the influence of cocoa on macrophages.

Some other studies have tried to improve CIA through flavonoid intake. In the same way it has been shown here, CIA mice treated subcutaneously with the flavonoid genistein do not exhibit a reduction in hind-paw swelling, although there is a decrease of the serum anti-CII antibody concentration ${ }^{(44)}$. On the other hand, other authors have reported the beneficial effect of isolated flavonoids or products containing flavonoids in CIA animals ${ }^{(45-50)}$. It should be pointed out, however, that some of these studies in CIA rats and mice do not find hindpaw swelling improvement after $27 \mathrm{~d}$ of induction but later, between after a month of induction and up to $53 \mathrm{~d}^{(46-48)}$. Recently, Cho et al. ${ }^{(51)}$ demonstrated the attenuation of CIA in mice by grape seed proanthocyanidins, but they provided these antioxidants by the intraperitoneal route and the improvement was evident later than 4 weeks after immunisation. All these results reassert the idea that a longer study could demonstrate clinically the beneficial effect of cocoa intake in the CIA rat model.

In conclusion, a cocoa diet in CIA rats involves a decrease in the main isotypes of specific anti-CII antibodies at 4 weeks of arthritis induction. Moreover, cocoa intake modulates the inflammatory response of macrophages, and decreases the Th:Tc ratio in ILN. However, these effects were not enough to reduce hind-paw swelling at the end of the study. Further studies could focus on the effect of a cocoa diet over a longer period of CIA and ascertain lymphocyte and macrophage cell function closer to inflamed joints. Anyway, a cocoa-enriched diet could be a good adjuvant therapy in disorders with oxidative stress or autoimmune pathogenesis.

\section{Acknowledgements}

The present study was supported by the Ministerio de Educación y Ciencia, Spain (AGL2005-002823) and by the Generalitat de Catalunya, Spain (SGR 2005-0083). S. R.-R. is the recipient of a fellowship from the Ministerio de Educación y Ciencia, Spain (BES-2006-13640). T. P.-B. is the recipient of a fellowship from the University of Barcelona. The authors declare they have no competing interests. S. R.-R. carried out the study, performed the statistical analysis and drafted the manuscript. F. J. P.-C., T. P.-B. and C. C. collaborated during the in vivo part and in the discussion of the results. M. C. and A. F. designed, supervised and coordinated the study. All the authors read and approved the final manuscript. The authors thank the 'Serveis Científico-Tècnics' of the University of Barcelona, especially Dr J. Comas, for expert assistance in flow cytometry, and Dr. Mónica Sauleda and Marta Bes (Banc de Sang, Hospital Vall d'Hebron, Barcelona) for the loan of the ELISPOT reader and their technical assistance. Moreover the authors thank E. Sanchez-Galan and D. Mis-Castell for their help in the ELISA method.

\section{References}

1. Firestein GS (2003) Evolving concepts of rheumatoid arthritis. Nature 423, 356-361.

2. Tobón GJ, Youinou P \& Saraux A (2010) The environment, geo-epidemiology, and autoimmune disease: Rheumatoid arthritis. J Autoimmun 35, 10-14.

3. Duskin A \& Eisenberg RA (2010) The role of antibodies in inflammatory arthritis. Immunol Rev 233, 112-125.

4. Kannan K, Ortmann RA \& Kimpel D (2005) Animal models of rheumatoid arthritis and their relevance to human disease. Pathophysiology 12, 167-181.

5. Holmdahl R, Lorentzen JC, Lu S, et al. (2001) Arthritis induced in rats with non-immunogenic adjuvants as models for rheumatoid arthritis. Immunol Rev 184, 184-202. 
6. Brand DD, Kang AH \& Rosloniec EF (2003) Immunopathogenesis of collagen arthritis. Springer Semin Immunopathol 25, 3-18.

7. Okamoto Y, Gotoh Y, Tokui H, et al. (2000) Characterization of the cytokine network at a single cell level in mice with collagen-induced arthritis using a dual color ELISPOT assay. J Interferon Cytokine Res 20, 55-61.

8. Wernhoff P, Unger C, Bajtner E, et al. (2001) Identification of conformation-dependent epitopes and $V$ gene selection in the $\mathrm{B}$ cell response to type II collagen in the DA rat. Int Immunol 13, 909-919.

9. Gomes A, Fernandes E, Lima JL, et al. (2008) Molecular mechanisms of anti-inflammatory activity mediated by flavonoids. Curr Med Chem 15, 1586-1605.

10. Lee KW, Kim YJ, Lee HJ, et al. (2003) Cocoa has more phenolic phytochemicals and a higher antioxidant capacity than teas and red wine. J Agric Food Chem 51, 7292-7295.

11. Ono K, Takahashi T, Kamei M, et al. (2003) Effects of an aqueous extract of cocoa on nitric oxide production of macrophages activated by lipopolysaccharide and interferongamma. Nutrition 19, 681-685.

12. Ramiro E, Franch A, Castellote C, et al. (2005) Flavonoids from Theobroma cacao down-regulate inflammatory mediators. J Agric Food Chem 53, 8506-8511.

13. Ramos-Romero S, Ramiro-Puig E, Pérez-Cano FJ, et al. (2008) Anti-inflammatory effects of cocoa in rat carrageenininduced paw oedema. Proc Nutr Soc 67, E65.

14. Castell M, Franch A, Ramos-Romero S, et al. (2009) Effect of a diet rich in cocoa flavonoids on experimental acute inflammation. In Flavonoids: Biosynthesis, Biological Effects and Dietary Sources, 1st ed., pp. 213-229 [RB Keller, editor]. Hauppauge: Nova Science Publishers, Inc.

15. Pérez-Berezo T, Ramiro-Puig E, Pérez-Cano FJ, et al. (2009) Influence of a cocoa-enriched diet on specific immune response in ovalbumin-sensitized rats. Mol Nutr Food Res 53, 389-397.

16. Ramiro-Puig E, Pérez-Cano FJ, Ramos-Romero S, et al. (2008) Intestinal immune system of young rats influenced by cocoaenriched diet. J Nutr Biochem 19, 555-565.

17. Reeves PG, Nielsen FH \& Fahey GC Jr (1993) AIN-93 purified diets for laboratory rodents: final report of the American Institute of Nutrition ad hoc writing committee on the reformulation of the AIN-76A rodent diet. J Nutr 123, 1939-1951.

18. Brahn E, Peacock DJ \& Banquerigo ML (1991) Suppression of collagen-induced arthritis by combination cyclosporin A and methotrexate therapy. Arthritis Rheum 34, 1282-1288.

19. Michaelsson E, Andersson M, Engstrom A, et al. (1992) Identification of an immunodominant type-II collagen peptide recognized by $\mathrm{T}$ cells in $\mathrm{H}-2(\mathrm{q})$ mice: self tolerance at the level of determinant selection. Eur J Immunol 22, 1819-1825.

20. Bajtner E, Nandakumar KS, Engström A, et al. (2005) Chronic development of collagen-induced arthritis is associated with arthritogenic antibodies against specific epitopes on type II collagen. Arthritis Res Ther 7, R1148-R1157.

21. Liu M, Wang X, Yin C, et al. (2007) Targeting TNF- $\alpha$ with a tetravalent mini-antibody TNF-TeAb. Biochem $J$ 406, $237-246$.

22. Cortés M, Canellada A, Miranda S, et al. (2008) Placental secreted factors: their role in the regulation of anti-CII antibodies and amelioration of collagen induced arthritis in rats. Immunol Lett 119, 42-48.

23. Du F, Lü LJ, Fu Q, et al. (2008) T-614, a novel immunomodulator, attenuates joint inflammation and articular damage in collagen-induced arthritis. Arthritis Res Ther 10, R136.
24. Zhao J, Li R, He J, et al. (2008) Mucosal administration of an altered CII263-272 peptide inhibits collagen-induced arthritis by suppression of Th1/Th17 cells and expansion of regulatory T cells. Rheumatol Int 29, 9-16.

25. Ramiro-Puig E, Pérez-Cano FJ, Ramírez-Santana C, et al. (2007) Spleen lymphocyte function modulated by a cocoaenriched diet. Clin Exp Immunol 149, 535-542.

26. Ramos-Romero S, Pérez-Berezo $\mathrm{T}$, Suàrez-Germà $\mathrm{C}$, et al. (2008) Influencia de una dieta rica en flavonoides sobre las poblaciones linfociticas durante un proceso artrítico experimental. Inmunología 27, S58-S59.

27. Brand DD, Marion TN, Myers LK, et al. (1996) Autoantibodies to murine type II collagen in collagen-induced arthritis: a comparison of susceptible and nonsusceptible strains. J Immunol 157, 5178-5184.

28. Stasiuk LM, Abehsira-Amar O \& Fournier C (1996) Collageninduced arthritis in $\mathrm{DBA} / 1$ mice: cytokine gene activation following immunization with type II collagen. Cell Immunol 173, 269-275.

29. Ye XJ, Tang B, Ma Z, et al. (2004) The roles of interleukin-18 in collagen-induced arthritis in the BB rat. Clin Exp Immunol 136, 440-447.

30. Saoudi A, Bernard I, Hoedemaekers A, et al. (1999) Experimental autoimmune myasthenia gravis may occur in the context of a polarized Th1- or Th2-type immune response in rats. J Immunol 162, 7189-7197.

31. Bridle BW, Wilkie BN, Jevnikar AM, et al. (2009) Steering orally induced deviation of xenogeneic immunity with exogenous cytokines in rats fed porcine proteins. Transpl Immunol 22, 32-37.

32. Ramos-Romero S, Pérez-Cano FJ, Pérez-Berezo $\mathrm{T}$, et al. (2010) A seven-day high cocoa diet decreases oxidant and inflammatory properties of peritoneal macrophages in rats. Proc Nutr Soc 69, E253.

33. Shakhov AN, Collart MA, Vassalli P, et al. (1990) Kappa B-type enhancers are involved in lipopolysaccharide-mediated transcriptional activation of the tumor necrosis factor alpha gene in primary macrophages. J Exp Med 171, 35-47.

34. Reagan-Shaw S, Nihal M \& Ahmad N (2008) Dose translation from animal to human studies revisited. FASEB $J \mathbf{2 2}$, 659-661.

35. Borchers AT, Keen CL, Hannum SM, et al. (2000) Cocoa and chocolate: composition, bioavailability, and health implications. J Med Food 3, 77-105.

36. Haskó G \& Cronstein B (2011) Methylxanthines and inflammatory cells. Handb Exp Pharmacol 200, 457-468.

37. Schley PD \& Field CJ (2002) The immune-enhancing effects of dietary fibres and prebiotics. Br J Nutr 87, S221-S230.

38. Wang F, Tan W, Guo D, et al. (2007) Reduction of CD4 positive $\mathrm{T}$ cells and improvement of pathological changes of collagen-induced arthritis by FTY720. Eur J Pharmacol 573, 230-240

39. Morgan ME, Flierman R, van Duivenvoorde LM, et al. (2005) Effective treatment of collagen-induced arthritis by adoptive transfer of CD25 + regulatory T cells. Arthritis Rheum $\mathbf{5 2}$ 2212-2221.

40. Kelchtermans H, Geboes L, Mitera T, et al. (2009) Activated $\mathrm{CD} 4+\mathrm{CD} 25+$ regulatory $\mathrm{T}$ cells inhibit osteoclastogenesis and collagen-induced arthritis. Ann Rheum Dis 68, 744-750.

41. Sarkar S \& Fox DA (2007) Regulatory T cell defects in rheumatoid arthritis. Arthritis Rheum 56, 710-713.

42. Stolina M, Bolon B, Dwyer D, et al. (2008) The evolving systemic and local biomarker milieu at different stages of disease progression in rat collagen-induced arthritis. Biomarkers 13, 692-712. 
43. Charles-Schoeman C, Banquerigo ML, Hama S, et al. (2008) Treatment with an apolipoprotein A-1 mimetic peptide in combination with pravastatin inhibits collagen-induced arthritis. Clin Immunol 127, 234-244.

44. Verdrengh M, Jonsson IM, Holmdahl R, et al. (2003) Genistein as an anti-inflammatory agent. Inflamm Res $\mathbf{5 2}$, 341-346.

45. Haqqi TM, Anthony DD, Gupta S, et al. (1999) Prevention of collagen-induced arthritis in mice by a polyphenolic fraction from green tea. Proc Natl Acad Sci $U S A$ 96, 4524-4529.

46. Kawaguchi K, Maruyama H, Kometani T, et al. (2006) Suppression of collagen-induced arthritis by oral administration of the citrus flavonoid hesperidin. Planta Med $\mathbf{7 2}$, $477-479$.

47. Imada K, Lin N, Liu C, et al. (2008) Nobiletin, a citrus polymethoxy flavonoid, suppresses gene expression and production of aggrecanases-1 and -2 in collagen-induced arthritic mice. Biochem Biophys Res Commun 373, 181-185.

48. Kometani T, Fukuda T, Kakuma T, et al. (2008) Effects of $\alpha$-glucosylhesperidin, a bioactive food material, on collageninduced arthritis in mice and rheumatoid arthritis in humans. Immunopharmacol Immunotoxicol 30, 117-134.

49. Miyake M, Ide K, Sasaki K, et al. (2008) Oral administration of highly oligomeric procyanidins of Jatoba reduces the severity of collagen-induced arthritis. Biosci Biotechnol Biochem 72, 1781-1788.

50. Dang DTN, Eriste E, Liepinsh E, et al. (2009) A novel antiinflammatory compound, artonkin-4'-O-glucoside, from the leaves of Artocarpus tonkinensis suppresses experimentally induced arthritis. Scand J Immunol 69, 110-118.

51. Cho ML, Heo YJ, Park MK, et al. (2009) Grape seed proanthocyanidin extract (GSPE) attenuates collageninduced arthritis. Immunol Lett 124, 102-110. 